\title{
HIGH RESOLUTION INFRARED MAPS OF IRAS GALAXIES
}

\author{
S.K. Ghosh, T.N. Rengarajan and R.P. Verma \\ Tata Institute of Fundamental Research \\ Homi Bhabha Road, Bombay 400 005, India
}

IRAS has provided infrared maps of a large number of galaxies. However the size of IRAS survey detectors is quite large $\left(\sim 5^{\prime}\right.$ in the cross-scan direction) and there is a need for improving the resolution of these maps. In order to understand the spatial structure of galaxies, we have generated high resolution maps of 18 large galaxies by deconvolving the IRAS pointed observations from survey detectors as we11 as from Chopped Photometric Channe1 (CPC) using Maximum Entropy Method (MEM).

The present sample of galaxies has been selected from "A catalog of IRAS observations of large optical galaxies" (LGC : Rice, W. et. al., Ap.J. Suppl., 68, 91, 1988). The LGC presents IRAS observations of 85 large galaxies (with blue-light major diameters greater than $8^{\prime}$ ), out of which 49 were observed in pointed mode with multi-scans for each galaxy. We have selected 18 galaxies which were observed in the pointed mode with DPS02B Macro with the angle between different scans for the same galaxy less than 2 degrees. We have deconvolved the data for each of the 4 IRAS survey bands (hereafter referred as AO) 12, 25, 60 and 100 $\mu \mathrm{m}$, as well as the CPC data at 50 and $100 \mu \mathrm{m}$. The point source functions were obtained from the respective $A O$ and $C P C$ observations of the planetary nebula NGC6543. The local average background as well as the noise for each map were estimated from the data in the immediate neighbourhood around the galaxy, for use during the deconvolutions.

We have obtained the deconvolved maps from AO for all the 18 sample galaxies and CPC maps for some of the galaxies. Deconvolved sizes at $10 \%$ level of a known point source are (in-scan x cross-scan): $0.7 \times 2.2$ $(12 \mu \mathrm{m} \& 25 \mu \mathrm{m}) ; 1 ! 2 \times 2 ! 5(60 \mu \mathrm{m}) ; \quad 3^{\prime} \times 3^{\prime}(100 \mu \mathrm{m})$ for A0s and $1^{\prime}$ dia $(50 \mu \mathrm{m})$ and $1^{\prime} .4$ dia $(100 \mu \mathrm{m})$ for CPC maps. The deconvolved maps of our sample of galaxies show consistent structures at the scale of $2-5^{\prime}$. The dynamic range for strong galaxies is $\sim 300$ for the $A 0$ and $\sim 20$ for the CPC. Many of these galaxies show multiple components. Almost all the galaxies show extended emission which is several times larger than the nuclear emission. On the average, the size of the galaxy along major axis (at 60 and $100 \mu \mathrm{m}$ ), at $2.5 \%$ contour level is about two thirds of the optical size. There is a very good correlation between $A 0$ and CPC structures. For illustration the deconvolved maps (AO and $C P C$ ) for NGC 891 are shown in Figure 1. 

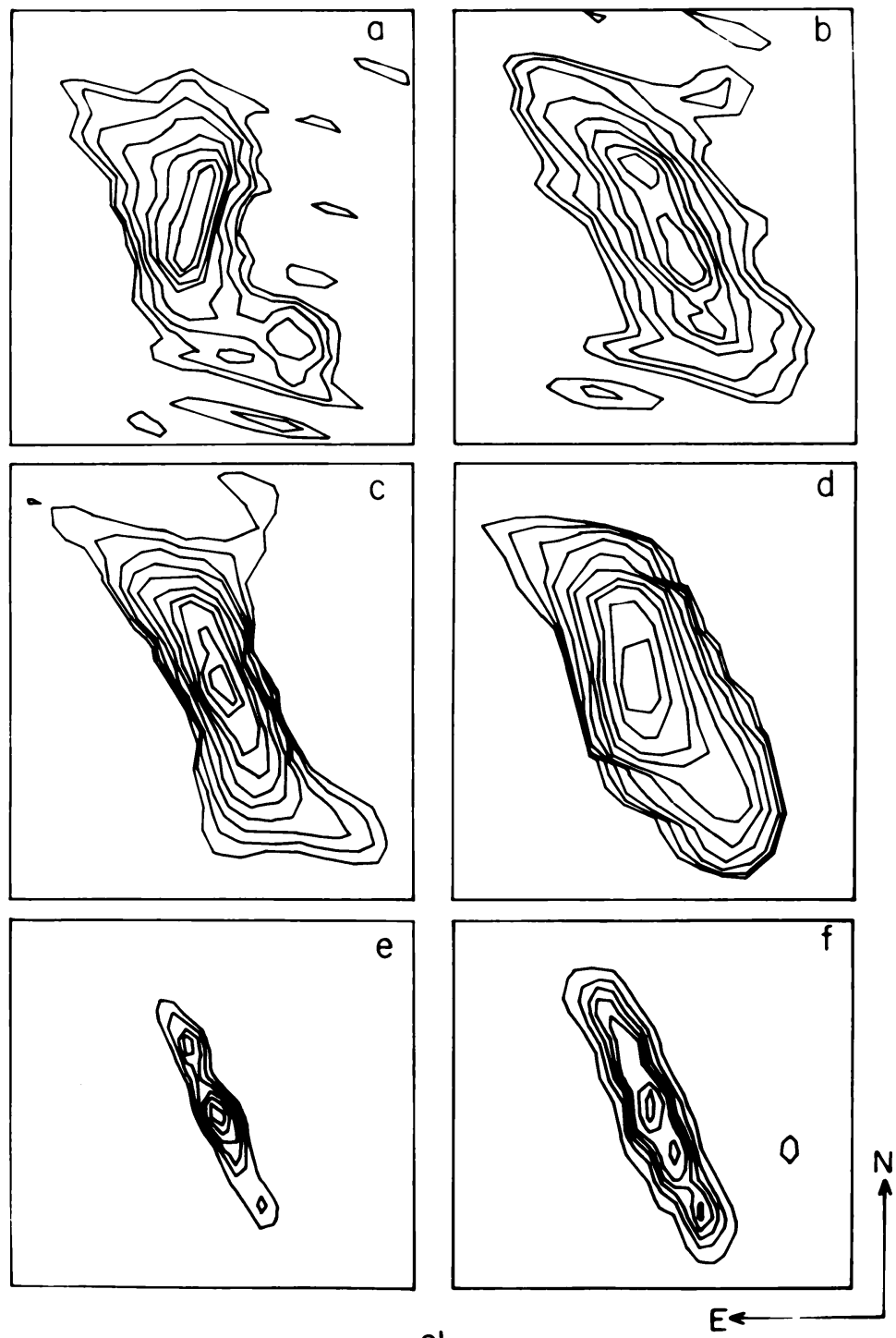

$12^{\prime}-1$

Fig. 1 Deconvolved IRAS maps at a) $12 \mu \mathrm{m}$, b) $25 \mu \mathrm{m}$, c) $60 \mu \mathrm{m}$, and d) $100 \mu \mathrm{m}$ from $A O$ and e) $50 \mu \mathrm{m}$ and f) $100 \mu \mathrm{m}$ from CPC. The contour levels (as percent of the peak) are a), b) $70,50,30,20$, $10,5,2.5$ and 1.25 ; c) and d) $70,50,30,20,10,5,2.5,1.25$, 0.63 and 0.31 ; e) $90,70,50,40,30$ and 20 ; f) $90,70,50,40$, 30,20 and 10 . The peak intensities in $\mathrm{Jy} /(\mathrm{sq} \cdot \operatorname{arc}$. min.) for a) to $f$ ) are $1.23,1.2,18.6,27.5,39.3$ and 75.8 res pectively. 